\title{
Pharmacists' Preferences and Choice of Essential Drugs: An Application of the Discrete Choice Experiment
}

\author{
Xuan Yu, Cheng Li, Xianzhi Zhang \& Min Yu (Corresponding author) \\ Department of Health Services, The Fourth Military Medical University \\ 17 ChengleXi Road, Xi'an 710032, China \\ Tel: 86-29-8477-4854 E-mail:myu@fmmu.edu.cn
}

Jin Zhang

School of Stomatology, The Fourth Military Medical University

145 ChengleXi Road, Xi'an 710032, China

\begin{abstract}
Pharmacists take an important role in prescribing drugs and have great influence on the implementation of national drug policies. The aim of this article is to analyze the preferences and choice of essential drugs among pharmacists in China. A questionnaire survey, designed as a discrete choice experiment, was sent to 90 individuals. In the questionnaire, different scenarios were constructed on the basis of six attributes: disease prevalence, severity of disease, age of target group, route of administration, individual health benefits, and cost-effectiveness. Individual preferences and choice were regressed against those characteristics. Binary logistic regression model was established to determine the relative importance of the attributes. The results showed that all aspects proved to significantly influence the respondents' preferences.
\end{abstract}

Keywords: Essential drug, Discrete choice experiment, Preference

\section{Introduction}

The essential drug concept was advocated by the WHO in 1977 in order to advise member states on the selection and procurement at reasonable cost of essential drugs of established quality corresponding to their national needs (WHO, 1995). Essential drugs are defined as those that satisfy the health care needs of the majority of the population, at a price they and the community can afford; they should therefore be available at all times and in adequate amounts, and in appropriate dosage forms (WHO, 1999). Therefore, a national list of essential drugs reflects public health priorities and helps a government to define its national priorities for public procurement and reimbursement and for training health professionals. The WHO issues the Model Essential Drug List (EDL) which is updated every 2 years and which serves as a model which governments adapt to respond to their national health priorities.

Currently, the Essential Drugs List forms an integral part of national drug policies in most WHO member states, guiding the selection of drugs on the basis of public health relevance, efficacy, safety and cost. However the implementation of an effective essential drug program is still meeting serious problems. China is no exception.

Since 1982, the national essential drugs list has been revised six times and the last time was in 2009, which is presently used as the base for all patients' prescriptions for those enrolled in the Basic Health Insurance Schemes. The health insurance authorities have adopted essential drug lists, which excluded some expensive and imported drugs that limited the scope of drugs that doctors can prescribe, to contain the escalation of drug expenditure and to improve the rational use of drugs through economically indirect restriction of prescription drugs. However, the decision to list an item on the formulary is not transparent nor does it consider the cost-effectiveness consideration. The effect of the drug lists combined with coverage of health insurance was to exaggerate the problem of over-prescription, which results in the growth of pharmaceutical expenditure.

Many essential drugs are not being manufactured because of the low financial returns or no benefits. The availability of essential drugs within China's market is considered very low; the median availability of generic medicines, in Shanghai in 2006, reached only to 33.3\% among the public facilities (Ye, 2006).

In recent years, China has further implemented a number of policies with the aim to ensure appropriate access to essential drugs including: campaigns for promoting the prescription of essential drugs, designating national producers of essential drugs to guarantee manufacturing of essential drugs (SFDA, 2006), organizing essential 
drugs bulk procurements methods and established a centralized distribution system to overcome the shortages of supplies (NDRC \& MOH, 2006). The accessibility of essential drugs is considered as a major problem. (Yan et al., 2009)

This indicates the need for rational approaches to the selection of essential drugs. However, the selection of essential drugs is a multi-criteria decision process. Following the experience of multiple-criteria approach in environment, (Linkov et al., 2004) and marketing (McDaniel and Gates, 2005) science, we aim to develop a similar approach to explore the pharmacists' preferences and choice of essential drugs. It provides a rational way to select essential drugs.

\section{Methods}

\subsection{Questionnaire design}

To elicit meaningful attributes for discrete choice experiment, an open questionnaire, literature review and expert interviews were used to identify each factor. Then two rounds of focus groups which included a total of six policy makers, academies, medical doctors and pharmacists were organized to identify the relevant criteria and related levels to be included in the discrete choice experiment.

Six attributes were included in the discrete choice experiment: disease prevalence, severity of disease, age of target group, route of administration, individual health benefits, and cost-effectiveness. (Zhang, 2009) The levels assigned to these attributes were: not prevalent or prevalent, not severe or severe, young, middle or elderly age group, convenient or inconvenient routes of administration, small or large individual health benefits, and non cost-effective or cost-effective.

The levels chosen for disease prevalence, severity of disease, route of administration, individual health benefits, and cost-effectiveness were qualitative. The levels of age of target group were in line with the concept of age-weighting. (Murray and Lopez, 1997)

Based on five criteria measured at two levels, and one criterion at three levels, a total number of 48 unique scenarios can be defined for inclusion in a full factorial experimental design in the discrete choice experiment. This number of combinations exceeds in practice what an individual could handle in a personal interview. Thus, a technique that allows researchers to reduce this number to a reasonable level without compromising statistical efficiency needs to be employed. However, random selection of discrete choices could yield a set of choices representing a small range of implied discount rates. To tackle this issue, a balanced incomplete block design was used to bring the number of choices for each participant down to a more manageable level of 16 choices. (Ryan and Gerard, 2003)

Each of these 16 scenarios was paired to its mirror image so as to retrieve the maximum information from each choice. An example of a pair of choices is given in Table 2.

Respondents were asked to 'imagine' they were currently making a decision between two different drugs. One of them should be included as essential drug.

\subsection{Statistical model}

In this study, essential drugs are described in terms of six key characteristics. Using random utility, a respondent's decision-making process during the completion of the questionnaire is modeled as the difference between two indirect utility functions where each utility function is that associated with a different drug. The respondents make a series of pair-wise choices and for each they choose the drug that leads to the higher level of utility. Thus the respondent would choose drug A over B if:

$$
U\left(A_{A}, Z\right)>U\left(A_{B}, Z\right)
$$

Where $\mathrm{U}$ represents the respondent's indirect utility function from drugs, $A_{A}$ the characteristics of drug A, $A_{B}$ the characteristics of drug $\mathrm{B}$ and $\mathrm{Z}$ socio-economic characteristics of the respondent that influence his/her preferences.

The respondent is assumed to know the nature of the utility function. This introduces the concept of random utility, where an error term is included in the utility function to reflect the unobservable factors in the individual's utility function. Thus, within the random utility framework, the respondent will choose A over B if:

$$
V\left(A_{A}, Z\right)+\varepsilon_{A}>V\left(A_{B}, Z\right)+\varepsilon_{B}
$$

where $\mathrm{V}$ is the measurable component of utility estimated empirically and $A_{A}, A_{B}$ and $\mathrm{Z}$ are as defined above 
and $\varepsilon_{j}(\mathrm{j}=\mathrm{A}, \mathrm{B})$ reflects the unobservable factors in the individual's utility function. Assuming a linear utility function $\mathrm{V}$, the utility to be estimated in pair-wise choice questions in moving from drug $\mathrm{B}$ to $\mathrm{A}$ is:

$$
\Delta V=\left\{\alpha_{0 A}+\sum \alpha_{i A} A_{i A}+Z+\varepsilon_{A}\right\}-\left\{\alpha_{0 \mathbf{B}}+\sum \alpha_{i B} A_{i B}+Z+\varepsilon_{B}\right\}
$$

which can be simplified as:

$$
\Delta V=\alpha_{0 A}-\alpha_{0 B}+\sum \beta_{i} A_{i}+e
$$

where $\alpha_{0 A}-\alpha_{0 B}$ is the constant term in the model, reflecting the overall preference for A over B when there is no difference between the levels of characteristics across drugs A and $\mathrm{B}, \sum \beta_{i}$ represent the parameters of the model to be estimated and $e$ is the unobservable factors in the utility function.

Further, assuming a linear model and given that the random utility model involves a comparison of two indirect utility functions, terms common to both utility functions will drop out of the measurable part of the utility function. Thus, $\mathrm{Z}$ will drop out. A binary logistic regression model which is linear in personal characteristics $(\mathrm{Z})$ leads to restrictive assumptions about the parameters to be estimated. More specifically, nonrandom variation in the coefficients is not allowed for. However, respondents' preferences may vary systematically with their personal characteristics. To allow for such nonrandom variation in preferences, the model can be segmented according to these characteristics. (Ryan, 1999)

\section{Results}

Sixty five of the ninety hospital pharmacists responded within the required two weeks time scale, giving a response rate of $72.2 \% .56 \%$ of them were male. The mean average age of respondents was 37 years, the youngest was 28 and the oldest 55 . The mean average working time was 19 years, the shortest was 5 and the longest was 37 .

The results from the random effects regression equation are shown in Table 3 for consistent responders. All coefficients have the expected direction and all are significant at the $1 \%$ level. This means that the respondents were considering all of the dimensions in their decision-making process. This is what we would expect as the dimensions were identified from focus group discussions. These results also support the internal validity of discrete choice experiment.

The OR shows, for example, that odds of being select as essential drugs is 1.9 time higher with the drugs that target prevalent diseases compared to not prevalent ones, other things being equal. Also, drugs that target severe diseases are almost five times probability of being selected than drugs that target the non severe diseases. Overall, drugs that target prevalent, severe disease, people of middle aged group, have large individual health benefits and are convenient of administration and cost-effective have a higher probability of being selected as essential drugs than drugs without those characteristics.

The model explained $35 \%$ of all observed variance in stated preference.

\section{Conclusion}

This research has shown how the pharmacists take into account multiple criteria simultaneously when they make choices. Essential drugs will be much more dispensed if policy makers concerned disease prevalence, severity of disease, age of target group, route of administration, individual health benefits, and cost-effectiveness. As a method of multiple-criteria decision analysis, discrete choice experiment can be employed to avoid concentrating on single criteria and to integrate and judge the relative importance of each criterion.

This research also raised a number of issues, some of which will be addressed in future work. Pharmaceutical policy development is a challenge for governments and health-care systems. The issues are complex, and there are many stakeholders with various social, economic, and political interests. In term of the selection of essential drugs, further research need to be conducted among policy makers, doctors, and patients to explore the difference of their preferences. Then a rational and transparent approach should be developed to select essential drugs.

\section{References}

Linkov, I., Varghese, A., \& Jamil, S. (2004). Multi-criteria decision analysis: a framework for structuring remedial decisions at contaminated sites. Comparative risk assessment and environmental decision making Amsterdam, 15-54. 
Murray, C. J. \& Lopez, A.D. (1997). Alternative projections of mortality and disability by cause 1990-2020: Global Burden of Disease Study. The Lancet 349, 1498-1504.

McDaniel, C. \& Gates, R. (2005). Marketing research essentials. (5th edition.). Hoboken, NJ: John Wiley \& Sons Inc.

National Development and Reform Commission (NDRC) \& Ministry of Health (MOH), China. (2006). Strengthening the administration of prices for the health services and medicines in urban community health service facilities. [Online] Available http://www.moh.gov.cn/newshtml/12673.htm (July 25, 2009)

Ryan, M. \& Gerard, K. (2003). Using discrete choice experiments to value health care programmes: current practice and future research reflections. Applied Health Economics and Health Policy, 2, 55-64.

Ryan, M. (1999). Using conjoint analysis to go beyond health outcomes: an application to in vitro fertilisation. Social Science \& Medicine, 48, 535-546.

State of Food and Drug Administration (SFDA), China. (2006). The first list of names about the designated national producers for essential medicines within urban communities and rural areas. [Online] Available http://www.sfda.gov.cn/WS01/CL0055/10641.html (August 31, 2009)

World Health Organization (WHO), Geneva. (1995). The Use of Essential Drugs. Sixth Report of the WHO Expert Committee. In: Technical Report Series, No. 850.

World Health Organization (WHO), Geneva. (1999). Report of the WHO Expert Committee on the Use of Essential Drugs.

Yan, J.J., Gao, W.Y., \& Zhang, B.L. (2009). Selection of national essential drugs based on analytic hierarchy process. Chinese Rural Health Service Administration, 2, 132-134.

Ye, L. (2006). ASurvey of Medicine Prices, Availability and Affordability in Shanghai, China applying the WHO/HAI Methodology. Fudan University, 20-21.

Zhang, W.Y. (2009). Introduction of Pharmacoeconomic Evaluation into the Selection of National Essential Drugs. China Pharmacy, 20, 561-562.

Table 1. Attributes and levels included in the study

\begin{tabular}{lcll}
\hline Attribute & Regression variables & Levels & Coding for regression \\
\hline \multirow{2}{*}{ Disease prevalence } & $\mathrm{X} 1$ & Not prevalent & 0 \\
\multirow{2}{*}{ Severity of disease } & $\mathrm{X} 2$ & Prevalent & 1 \\
& & Not severe & 0 \\
Age of target group & (Middle aged)X3 & Young & 1 \\
& (Elderly)X4 & Middle age & 0,0 \\
Route of administration & & Elderly & 1,0 \\
Individual health & $\mathrm{X} 5$ & Convenient & 0,1 \\
benefits & $\mathrm{X} 6$ & Inconvenient & 0 \\
Cost-effectiveness & & Small & 1 \\
& $\mathrm{X} 7$ & Large & 0 \\
& & Not cost-effectiveness & 0 \\
& & Cost-effectiveness & 1 \\
\hline
\end{tabular}


Table 2. Example of choice

\begin{tabular}{|l|l|l|}
\hline Choice 1 & Drug A & Drug B \\
\hline Disease prevalence & Sever. & Not sever. \\
\hline Severity of disease & Not prevalent. & Prevalent. \\
\hline Age of target group & Young. & Elderly. \\
\hline Route of administration & Convenient. & Inconvenient. \\
\hline Individual health benefits & Small. & Large. \\
\hline Cost-effectiveness & Not cost-effectiveness. & Cost-effectiveness. \\
\hline Which drug would you prefer? (Tick one only.) & & \\
\hline
\end{tabular}

Table 3. Results from binary logistic regression model

\begin{tabular}{lrrrrr}
\hline Criteria & Coefficient & \multicolumn{1}{l}{ Sig. } & OR & \multicolumn{2}{c}{$95 \%$ CI } \\
\hline X1 & 0.648 & 0.006 & 1.912 & 1.208 & 3.026 \\
X2 & 1.666 & 0.000 & 5.292 & 3.312 & 8.456 \\
X3 & 1.094 & 0.000 & 2.987 & 1.684 & 5.299 \\
X4 & 0.831 & 0.003 & 2.296 & 1.315 & 4.011 \\
X5 & 1.125 & 0.000 & 3.082 & 1.991 & 4.770 \\
X6 & 0.812 & 0.002 & 2.253 & 1.360 & 3.731 \\
X7 & 0.564 & 0.007 & 1.758 & 1.131 & 2.732 \\
Constant & -3.243 & 0.000 & 0.039 & & \\
\hline
\end{tabular}

Nagelkerke R Square $=0.354$ 